Göteborg-ITP-96-8

hep-th/9606173

June, 1996
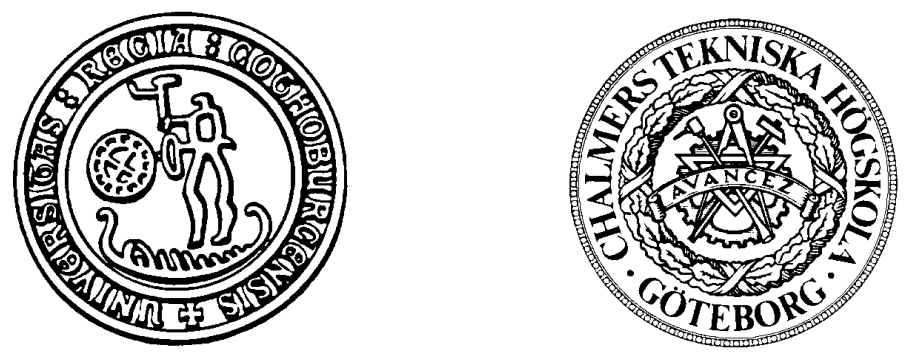

\title{
On the DiRAC-BORn-InfEld ACtion For D-BRAnes
}

\author{
Martin Cederwall, Alexander von Gussich, \\ Aleksandar Miković*, Bengt E.W. Nilsson and Anders Westerberg \\ Institute of Theoretical Physics \\ Göteborg University and Chalmers University of Technology \\ S-412 96 Göteborg, Sweden
}

Abstract: In this note, we consider the reformulation of the Dirac-Born-Infeld action for a Dirichlet p-brane in Brink-Di Vecchia-Howe-Tucker form, i.e., including an independent non-propagating world-volume metric. When $p>2$, the action becomes non-polynomial. A closed expression is derived for $p=3$. For selfdual field-strengths, the DBI action is reproduced by an action with a simple $F^{2}$ term. We speculate on supersymmetrization of the $\mathrm{D}_{3}$-brane action. We also give the governing equations for arbitrary $p$, and derive an implicit expression for the $\mathrm{D}_{4}$-brane lagrangian.

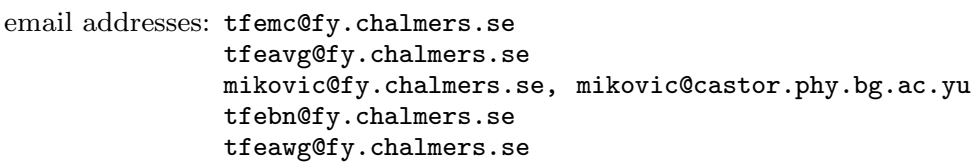

*Permanent address: Institute of Physics, P.O. Box 57, Belgrade 11001, Yugoslavia. 
The introduction of $p$-branes in string theory has turned out to be instrumental in unraveling many of its non-perturbative properties (see e.g. $[1,2,3]$ ). In type IIA and IIB string theory, $p$ branes for various values of $p$ arise as solutions of the low-energy field equations. They are of two kinds depending on whether the field equations of which they are solutions involve antisymmetric tensor fields from the NS-NS or the R-R sector. In the case of NS-NS fields, the $p$-branes are interpreted as either fundamental or solitonic in the sense familiar from ordinary field theory (see e.g. $[4,1])$. In the R-R sector, on the other hand, the $p$-branes are neither fundamental nor genuinely solitonic, but have been found to have a description in terms of open strings with mixed Dirichlet and Neumann boundary conditions, ending at the location of the $p$-branes $[5,6,7] \cdot p$-branes of this type are referred to as Dirichlet branes, or D-branes. More recently, also the 5-brane solution [8] of 11-dimensional supergravity has been given a similar interpretation in terms of open membranes [9].

The fact that $p$-branes with R-R charges are located at the ends of open strings implies that the $p$-brane world-volume field theory can be derived from open string $\beta$-functions [6]. For an abelian and constant field-strength $F_{m n}$, the result is a "kinetic" term of the Dirac-Born-Infeld (DBI) type.

The action for the $\mathrm{D}_{2}$-brane is known to be equivalent $[10,9]$ to the 11-dimensional supermembrane [11], which is the world-volume metric formulation of Howe and Tucker [12] generalizing the Brink-Di Vecchia-Howe string action [13] to $p \geq 2$ (we refer to the form of the action containing the world-volume metric as the Brink-Di Vecchia-Howe-Tucker (BDHT) form). In three dimensions, a vector potential is dual to a scalar, which can be interpreted as the eleventh coordinate [14]. Therefore, also the $\kappa$-symmetric version is known $[14,11,9]$. For higher values of $p$, no exact BDHT-type actions for D-branes have been constructed so far, although the action to lowest order in derivatives is given in [9], and only the bosonic parts of the DBI actions, including couplings to background fields, are known $[15,16]$. It is conceivable, and this is one of the main motivations for the present work, that the construction of supersymmetric $\mathrm{D}_{p}$-brane actions will benefit from knowledge of a BDHT-type action.

The purpose of this note is to develop techniques that may be useful in establishing links between the DBI forms of D-brane actions and their corresponding BDHT forms. As emphasized by Townsend [9], these latter forms are not likely to be simple for $p>2$, but, rather, become nonpolynomial in the field-strength $F$. In fact, the highly non-linear equations arising when varying with respect to the world-volume metric have not been solved previously for $p>1$ (although the $p=2$ solution is implicit in [9]). For higher values of $p$, not having general techniques for solving these equations also means that the higher order terms in the BDHT formulation can not be deduced.

The methods developed here indicate that these problematic issues can in fact be resolved. We will in particular demonstrate that, for $p=3$, the DBI action is equivalent to a BDHT action, which is non-polynomial, but of a reasonably simple form. A remarkable simplification occurs if a self-duality constraint is imposed; the action then becomes bilinear in $F$. It is also interesting to note that although the world-volume metric is not set equal to the induced metric by its equation of motion, their determinants are equal, a fact that may prove very useful. Other aspects of $\mathrm{D}_{3}$-brane actions have been addressed previously in $[17,18]$.

Consider a general lagrangian for a $p$-brane with an abelian vector potential ${ }^{1}$ :

$$
\mathscr{L}=-\frac{1}{2} \sqrt{-\gamma}\left\{\operatorname{tr}\left(\gamma^{-1} g\right)+\varphi\left(\gamma^{-1} \hat{F}\right)-(p-1)\right\}
$$

\footnotetext{
${ }^{1}$ We omit the dilaton factor $e^{-\phi}$, which is irrelevant for our discussion. Also, couplings to R-R background fields are not considered, since they enter as Wess-Zumino terms, not containing the world-volume metric.
} 
Here $\gamma_{m n}$ is the world-volume metric, $g_{m n}=\partial_{m} X^{\mu} \partial_{n} X^{\nu} G_{\mu \nu}$ the induced metric (i.e., the pullback of the space-time metric) and $\hat{F}_{m n}=F_{m n}-\frac{2 \pi}{\alpha^{\prime}} B_{m n}$ where $F_{m n}$ is the abelian field-strength and $B_{m n}$ the pull-back to the world-volume of the NS-NS antisymmetric tensor field. The function $\varphi$ is some scalar function of $\gamma^{-1} \hat{F}$, that should be chosen so that solving the equations of motion for $\gamma$ results in the Dirac-Born-Infeld lagrangian

$$
\mathscr{L}_{\mathrm{DBI}}=-\sqrt{-\operatorname{det}(g+\hat{F})} \text {. }
$$

As a warmup exercise, and in order to establish our procedure, we first treat the case $p=2$. We define the matrices

$$
\begin{aligned}
& u=\gamma^{-1} g, \\
& X=g^{-1} \hat{F},
\end{aligned}
$$

and the objective is to use the equations of motion for the world-volume metric in order to solve for $u$ in terms of $X$. In three dimensions, the matrix $X$ obeys the identity

$$
X^{3}=\frac{1}{2} X \operatorname{tr} X^{2}
$$

The only invariant to enter the lagrangian through $\varphi$ is $\operatorname{tr}(u X)^{2}$, so we can set $\varphi=\varphi\left(\operatorname{tr}(u X)^{2}\right)$. The equations of motion for $\gamma^{-1}$ become

$$
0=-\frac{2}{\sqrt{-\gamma}} \gamma^{-1} \frac{\partial \mathscr{L}}{\partial \gamma^{-1}}=-\frac{1}{2} \operatorname{tr} u-\frac{1}{2} \varphi+\frac{1}{2}+u+2(u X)^{2} \varphi^{\prime}
$$

It is clear that $u$ only contains even powers of $X$, and a convenient basis turns out to be $u=$ $A+B\left(X^{2}-\frac{1}{2} \operatorname{tr} X^{2}\right)$, where $A$ and $B$ are functions of $\operatorname{tr} X^{2}$. This turns the equations of motion (5) into

$$
0=\frac{1}{2}-\frac{1}{2} A-\frac{1}{4} B \operatorname{tr} X^{2}-\frac{1}{2} \varphi\left(A^{2} \operatorname{tr} X^{2}\right)+X^{2}\left[2 A^{2} \varphi^{\prime}\left(A^{2} \operatorname{tr} X^{2}\right)+B\right] .
$$

When $\varphi$ is just the ordinary $\hat{F}^{2}$ term, $\varphi(t)=-\frac{1}{2} t$, the solution is $A=B=1$. We insert the solution back into $\mathscr{L}$, using $\operatorname{det} u=\operatorname{det}\left(1+X^{2}-\frac{1}{2} \operatorname{tr} X^{2}\right)=1-\frac{1}{2} \operatorname{tr} X^{2}=\operatorname{det}(1+X)$ to obtain

$$
\begin{aligned}
\mathscr{L} & =-\sqrt{-\gamma}\left(1-\frac{1}{2} \operatorname{tr} X^{2}\right)=-\sqrt{-g}(\operatorname{det} u)^{-1 / 2}\left(1-\frac{1}{2} \operatorname{tr} X^{2}\right) \\
& =-\sqrt{-g} \sqrt{\operatorname{det}(1+X)}=-\sqrt{-\operatorname{det}(g+\hat{F})}
\end{aligned}
$$

and the equivalence to the Dirac-Born-Infeld action is established.

We now move on to $p=3$. The relevant matrix identity is

$$
X^{4}=\frac{1}{2} X^{2} \operatorname{tr} X^{2}-\operatorname{det} X
$$

The function $\varphi$ will depend on the invariants $\operatorname{tr}(u X)^{2}$ and $\operatorname{det}(u X)$, and $u$ can again be expanded as, e.g., $u=A+B X^{2}$. It turns out to be more convenient, however, to choose another basis, namely one where $X^{2}$ acts diagonally. From (8) it follows that

$$
X^{2} v_{ \pm}=\lambda_{ \pm} v_{ \pm}
$$


where

$$
\begin{aligned}
& \lambda_{ \pm}=\frac{1}{4} \operatorname{tr} X^{2} \pm \sqrt{-\Delta} \\
& v_{ \pm}=\frac{1}{2}\left[1 \pm \frac{1}{\sqrt{-\Delta}}\left(X^{2}-\frac{1}{4} \operatorname{tr} X^{2}\right)\right] .
\end{aligned}
$$

The scalar $\Delta$, that will be of some interest later, is defined as $\Delta(X)=\operatorname{det} X-\frac{1}{16}\left(\operatorname{tr} X^{2}\right)^{2}$. It is negative semi-definite. The matrices $v_{ \pm}$are normalized to become projection operators on the subspaces with the associated eigenvalues. We now expand $u$ as $u=u_{+} v_{+}+u_{-} v_{-}$, and obtain

$$
\begin{aligned}
& \operatorname{tr}(u X)^{2}=2\left(\lambda_{+} u_{+}^{2}+\lambda_{-} u_{-}^{2}\right) \\
& \operatorname{det}(u X)=\lambda_{+} \lambda_{-} u_{+}^{2} u_{-}^{2},
\end{aligned}
$$

so that the function $\varphi$ in the lagrangian (1) is a function of $\lambda_{+} u_{+}^{2}$ and $\lambda_{-} u_{-}^{2}$. After some manipulations, the equations of motion for $\gamma^{-1}$ become

$$
\begin{aligned}
& 1-\frac{1}{2} \varphi-u_{-}+\frac{1}{2} u_{+} \frac{\partial \varphi}{\partial u_{+}}=0 \\
& 1-\frac{1}{2} \varphi-u_{+}+\frac{1}{2} u_{-} \frac{\partial \varphi}{\partial u_{-}}=0 .
\end{aligned}
$$

The occurrence of derivatives should not be interpreted as if these equations were differential equations - for a given function $\varphi$ they are simply algebraic equations for $u_{ \pm}$. If, in addition, we want the action to be equivalent to the Dirac-Born-Infeld action, we use $\operatorname{det}(1+X)=\left(1-\lambda_{+}\right)\left(1-\lambda_{-}\right)$ to obtain the condition

$$
1-u_{+}-u_{-}-\frac{1}{2} \varphi+u_{+} u_{-} \sqrt{\left(1-\lambda_{+}\right)\left(1-\lambda_{-}\right)}=0
$$

The resulting set of equations is seemingly difficult to solve. After solving for $\varphi$ and $u_{ \pm}$order by order a couple of steps, we noticed that we got $\operatorname{det} u \equiv u_{+}^{2} u_{-}^{2}=1$, i.e., $\operatorname{det} \gamma=\operatorname{det} g$. We do not understand why this happens, but by assuming it to hold exactly one can considerably restrict the possible functions $\varphi$ by demanding them to give solutions with $\operatorname{det} u=1$ (this will be verified by the exact solution). The search is simplified by the change of variables to $s_{ \pm}=\left(2 \lambda_{ \pm} u_{ \pm}^{2}\right)^{-1}$, and $\chi=2\left(s_{+} s_{-}\right)^{1 / 2}\left(1-\frac{1}{2} \varphi\right)$. The determinant condition then becomes

$$
\frac{\partial \chi}{\partial s_{+}} \frac{\partial \chi}{\partial s_{-}}=1
$$

and the equations of motion (12) together with the condition (13) turn into

$$
\begin{gathered}
\frac{\partial \chi}{\partial s_{+}}-\frac{1}{\sqrt{2 \lambda_{-} s_{+}}}=0, \\
\sqrt{\lambda_{+} \lambda_{-}} \chi-\frac{1}{\sqrt{2 \lambda_{+} s_{+}}}-\sqrt{2 \lambda_{+} s_{+}}+\sqrt{\left(1-\lambda_{+}\right)\left(1-\lambda_{-}\right)}=0 .
\end{gathered}
$$

It is not difficult to find a number of functions satisfying (14), and if the system is going to be soluble, we can not afford complicated functions leading to transcendental equations. The simplest 
reasonable function is $\chi=2\left(s_{+} s_{-}\right)^{1 / 2}$, which of course corresponds to $\varphi=0$. We can modify it to $\chi=2\left(\left(s_{+}+\alpha\right)\left(s_{-}+\alpha\right)\right)^{1 / 2}$, and it turns out that the solution of $\left(15^{a}\right)$,

$$
\begin{aligned}
& s_{+}=\frac{1}{2 \lambda_{+}} \frac{1-2 \alpha \lambda_{+}}{1-2 \alpha \lambda_{-}}, \\
& s_{-}=\frac{1}{2 \lambda_{-}} \frac{1-2 \alpha \lambda_{-}}{1-2 \alpha \lambda_{+}},
\end{aligned}
$$

fulfills $\left(15^{b}\right)$ for $\alpha=\frac{1}{2}$. This gives the solution for the BDHT-type $\mathrm{D}_{3}$-brane lagrangian. Expressed in terms of the familiar variables $\operatorname{tr}(u X)^{2}$ and $\operatorname{det}(u X)$ the function $\varphi$ is

$$
\begin{aligned}
\varphi & =2\left\{1-\sqrt{\left(1+\lambda_{+} u_{+}^{2}\right)\left(1+\lambda_{-} u_{-}^{2}\right)}\right\} \\
& =2\left\{1-\sqrt{1+\frac{1}{2} \operatorname{tr}(u X)^{2}+\operatorname{det}(u X)}\right\} \\
& =2\left\{1-\sqrt{\left(1+\frac{1}{4} \operatorname{tr}(u X)^{2}\right)^{2}+\Delta(u X)}\right\} \\
& =2\left\{1-\left[\operatorname{det}\left(1+(u X)^{2}\right)\right]^{1 / 4}\right\},
\end{aligned}
$$

so the lagrangian reads

$$
\mathscr{L}=\sqrt{-\gamma}\left\{-\frac{1}{2} \operatorname{tr}\left(\gamma^{-1} g\right)+\sqrt{\left(1+\frac{1}{4} \operatorname{tr}\left(\gamma^{-1} \hat{F}\right)^{2}\right)^{2}+\Delta\left(\gamma^{-1} \hat{F}\right)}\right\}
$$

(note that the cosmological constant gets absorbed in the square root). The expression inside the square root is never negative. The occurrence of a square root should not be associated to the square root in the DBI action, it is rather a feature particular to $p=3$.

Notice that if $\Delta\left(\gamma^{-1} \hat{F}\right)$ vanishes, the lagrangian reduces to one containing only the $\hat{F}^{2}$ term, as for $p=2$. When we examine the meaning of this condition, we have to be careful about the signature of the world-volume metric. If it is lorentzian, as for the application we set out to investigate, both terms in $\Delta$ are negative semi-definite, so here it implies that $\operatorname{det}\left(\gamma^{-1} \hat{F}\right)=0=\operatorname{tr}\left(\gamma^{-1} \hat{F}\right)^{2}$, or equivalently, $\mathbb{E} \cdot \mathbb{B}=0=\mathbb{E}^{2}-\mathbb{B}^{2}$. Then all terms in $\mathscr{L}$ containing $\hat{F}$ vanish, and this case becomes trivial. If, on the other hand, the world-volume signature is $(4,0)$ or $(2,2)$, the condition is equivalent to selfduality or anti-selfduality (with respect to the world-volume metric). When we analyze this action, with a selfduality constraint, it turns out that it is irrelevant which metric (world-volume or induced) we refer to when selfduality is imposed. We can not use the basis (10), which is not well defined when $\Delta=0$, so we write $u=A+B\left(X^{2}-\frac{1}{4} \operatorname{tr} X^{2}\right)$, and the solution is straightforward, yielding $A=1, B=\left(1-\frac{1}{2} \operatorname{tr} X^{2}\right)^{-1}$. The Dirac-Born-Infeld lagrangian is again reproduced. We believe that this case may be relevant for the formulation of F-theory (see e.g. $[19,20,21])$.

There are a couple of questions that deserve further investigation. One is the generalization to higher $p$. It is not clear to us whether it will be possible to obtain closed forms for the BDHT-type lagrangians for $p>3$. It is conceivable that the equations simply become too complicated, but on the other hand, this was what we thought would happen already for $p=3$. 
The generalization of the method we have used for $p=3$ is straightforward, and probably gives the simplest formulation of the problem. For the case of odd $p, p=2 n-1$, there are $n$ non-zero eigenvalues $\lambda_{i}$ for $X^{2}$, each with an associated subspace of dimension two, $\operatorname{tr} v_{i}=2$. The equations of motion for $\gamma^{-1}$ together with the condition that the action reduces to the Dirac-Born-Infeld action read

$$
\begin{aligned}
& 0=n-1-\sum_{j \neq i} u_{j}-\frac{1}{2} \varphi+\frac{1}{2} u_{i} \frac{\partial \varphi}{\partial u_{i}}, \quad i=1, \ldots, n, \\
& \sum_{i} u_{i}+\frac{1}{2} \varphi-(n-1)=\prod_{i}\left(1-\lambda_{i}\right)^{1 / 2} u_{i} .
\end{aligned}
$$

For the case of even $p, p=2 n$, there are $n$ non-zero eigenvalues $\lambda_{i}$ for $X^{2}$, each with an associated linear subspace of dimension two, $\operatorname{tr} v_{i}=2$, and one zero eigenvalue with $\operatorname{tr} v_{0}=1$. The $v_{0}$ component of $u$ does not enter in $\varphi$, and can be solved for,

$$
u_{0}=-(2 n-1)+2 \sum_{i} u_{i}+\varphi
$$

The remaining equations read

$$
\begin{aligned}
& 0=-u_{0}+u_{i}+\frac{1}{2} u_{i} \frac{\partial \varphi}{\partial u_{i}}, \quad i=1, \ldots, n, \\
& u_{0}=\prod_{i}\left(1-\lambda_{i}\right) u_{i}^{2} .
\end{aligned}
$$

It seems difficult in general to determine $\varphi$ explicitly. For $p=4$ we have been able to find the explicit solution for $u$. Here we have two non-zero eigenvalues that we denote $\lambda_{ \pm}$, which expressed in terms of $X=\gamma^{-1} \hat{F}$ are $\lambda_{ \pm}=\frac{1}{4} \operatorname{tr} X^{2} \pm\left[\frac{1}{4} \operatorname{tr} X^{4}-\frac{1}{16}\left(\operatorname{tr} X^{2}\right)^{2}\right]^{1 / 2}$. The solution is

$$
u_{ \pm}=\left(1-\lambda_{ \pm}\right)^{-2 / 3}\left(1-\lambda_{\mp}\right)^{1 / 3} \text {. }
$$

The function $\varphi$, which is a function of $t_{ \pm}=\lambda_{ \pm} u_{ \pm}^{2}$, is then given implicitly by the algebraic equations

$$
\begin{aligned}
& t_{ \pm}=u_{ \pm}^{2}-\frac{1}{u_{\mp}}, \\
& \varphi=3+\frac{1}{u_{+} u_{-}}-2\left(u_{+}+u_{-}\right) .
\end{aligned}
$$

We have not yet been able to eliminate $u_{ \pm}$from these equations (note that solving for $u_{ \pm}$in terms of $t_{ \pm}$amounts to solving a fifth-order equation). There is a "miraculous identity" analogous to the identity $\operatorname{det} u=1$ for $p=3$, namely $u_{0} u_{+} u_{-}=1$ (here, however, $\operatorname{det} u \equiv u_{0} u_{+}^{2} u_{-}^{2}$ ). The occurrence of these properties of the solutions is intriguing.

We would also like to generalize to non-abelian field-strengths. It is not clear how to proceed in that case - the simple matrix identities we have utilized in the present note do not carry over.

A case of special interest is the 5-brane in eleven dimensions. Also in this case the methods we have presented here need modification, due to the presence of a 3 -form field-strength. The analogue of a DBI action is unknown for the 5-brane - it would require a generalization of the standard string $\beta$-function calculation to open membranes. 
Another urgent issue is supersymmetrization. One might think that the form of the lagrangian (18) would not present any advantages as compared to the Dirac-Born-Infeld action. However, one important thing happens that might help. When one looks for a $\kappa$-symmetric action, one needs a projection operator that splits a spinor in two equal parts - $\kappa$-symmetry is one half spinor worth of fermionic gauge symmetry, essential to reduce the number of physical fermionic degrees of freedom to the correct one. The solution we obtained for $p=3$ satisfied $\operatorname{det} u=1$ (for reasons we do not yet fully understand), which means that we may write down such a projection operator as $P=\frac{1}{2}(1+\Gamma)$, where

$$
\Gamma=\frac{1}{24 \sqrt{-\gamma}} \varepsilon^{k l m n} \Pi_{k}{ }^{k} \Pi_{l}{ }^{\lambda} \Pi_{m}{ }^{\mu} \Pi_{n}{ }^{\nu} \Gamma_{\kappa \lambda \mu \nu},
$$

where now $g_{m n}=\Pi_{m}{ }^{\mu} \Pi_{n \mu}$. The fact that $\operatorname{det} \gamma=\operatorname{det} g$ ensures that $\Gamma^{2}=1$. This observation makes the hope of finding a supersymmetric D-brane action for $p=3$ less far-fetched, even if the non-linearities may become difficult to deal with.

Finally, one should investigate the relevance of the case $\Delta=0$ to F-theory. It is striking that we have a very simple action for the case of selfdual field-strength. Although the constraint structure cannot be believed to be complete, one can hope to get some hints from this formulation, especially after supersymmetrization.

\section{REFERENCES}

[1] C.M. Hull and P.K. Townsend, "Unity of Superstring Dualities", Nucl.Phys. B438 (1995) 109 (hep-th/9410167).

[2] E. Witten, "String Theory Dynamics in Various Dimensions", Nucl.Phys. B443 (1995) 85 hep-th/9503124).

[3] M.J. Duff, "Strong/Weak Coupling Duality from the Dual String", Nucl.Phys. B442 (1995) 47 (hep-th/950103d).

[4] M.J. Duff, R.R. Khuri and J.X. Lu, "String Solitons", Phys.Rep. 259 (1995) 213 (hep-th/9412184).

[5] J. Dai, R.G. Leigh and J. Polchinski, "New Connections between String Theories", Mod.Phys.Lett. A4 (1989) 2073.

[6] R.G. Leigh, "Dirac-Born-Infeld Action from Dirichlet Sigma Model", Mod.Phys.Lett. A4 (1989) 2767 .

[7] J. Polchinski, "Dirichlet-Branes and Ramond-Ramond Charges", Phys.Rev.Lett. 75 (1995) 4724 (hep-th/9510017).

[8] R. Güven, "Black p-Brane Solutions of D=11 Supergravity Theory", Phys.Lett. 276B (1992) 49.

[9] P.K. Townsend, "D-branes from M-branes", Phys.Lett. 373B (1996) 68 (hep-th/9512062).

[10] C. Schmidhuber, "D-brane actions", hep-th/9601003.

[11] E. Bergshoeff, E. Sezgin and P.K. Townsend, "Properties of the Elevendimensional Supermembrane Theory", Ann.Phys. 185 (1988) 330.

[12] P.S. Howe and R.W. Tucker, "A Locally Supersymmetric and Reparametrization Invariant Action for a Spinning Membrane", J.Phys. A10 (1977) L155.

[13] L. Brink, P. Di Vecchia and P. Howe, "A Locally Supersymmetric and Reparametrization Invariant Action for the Spinning String", Phys.Lett. 65B (1976) 471.

[14] M.J. Duff and J.X. Lu, "Type II p-branes: the brane-scan revisited", Nucl.Phys. B390 (1993) 276 (hep-th/9207060).

[15] M. Douglas, "Branes within Branes", hep-th/9512077.

[16] M.B. Green, C.M. Hull and P.K. Townsend, "D-Brane Wess-Zumino Actions, T-Duality and the Cosmological Constant", hep-th/9604119.

[17] A.A. Tseytlin, "Self-duality of Born-Infeld action and Dirichlet 3-brane of type IIB superstring", hep-th/9602064.

[18] D.P. Jatkar and S.K. Rama, "F-theory from Dirichlet 3-branes", hep-th/9606009.

[19] M.P. Blencowe and M.J. Duff, "Supermembranes and the Signature of Space-Time", Nucl.Phys. B310 (1988) 387.

[20] C. Vafa, "Evidence for F-Theory", hep-th/9602022.

[21] C.M. Hull, "String Dynamics at Strong Coupling", hep-th/9512181. 